\title{
Trends in Selective Laser Sintering in Biomedical Engineering
}

\author{
Sanjeet.Chandra ${ }^{1,}$, S.H. Masood ${ }^{2}$, Syed Riza $^{3}$ \\ ${ }^{1,2,3}$ Faculty of Science, Engineering and Technology, Swinburne University of Technology, \\ Victoria, Australia 3122 \\ ${ }^{1}$ schandra@ swin.edu.au, ${ }^{2}$ smasood@ swin.edu.au, ${ }^{3}$ sriza@ swin.edu.au
}

\begin{abstract}
Selective Laser Sintering (SLS) has been introduced in early nineties as layer by layer additive manufacturing technology using laser beam to sinter the powder on a powder bed. Since then extensive research has been conducted in regards to its applications and the processing of materials. The materials used include mostly polymers, ceramics and composites. Major applications of SLS have been in development of industrial parts for biomedical and aerospace sectors, functional prototypes and patterns for casting. The focus of this paper is to present a brief overview and trends in the development of materials for SLS for biomedical applications up to the present time. Finding new materials in powder form that can withstand laser processing parameters and exhibit biocompatible properties are some of the future works required to assist in expanding SLS application in biomedical engineering field
\end{abstract}

Key words : About four key words or phrases in alphabetical order, separated by commas.

\section{INTRODUCTION}

Selective laser sintering (SLS) additive manufacturing technology involves the manufacturing of solid parts by fusing the powder layer by layer on a powder bed by a scanning laser beam. The effect of laser radiation causes preheated powder layer to go through the phase transformation. SLS is used for generating complex 3D parts directly from computer model while the parts are manufactured by strengthening the successive layers of powder on the top of each other. As shown in Figure 1, the strength is gained by sintering the successive powder layers using thermal energy, which is supplied by the laser beam to the powder deposition system. A successive thin layer between 0.1 to $0.3 \mathrm{~mm}$ thick is deposited on a platform before the layer gets sintered with the laser beam. The fabrication chamber is sealed for maintaining the temperature below the melting point of the powder $[1,2]$. These powders which are slightly compacted can be of particle sizes ranging from several microns to several hundred microns. There are other considerations of the powder for laser sintering such as shape and the morphology as these properties create an impact on the density of the powder bed and the flowability of the powder. The flowability of the powder is critical because the powder should be uniformly spread on the bed at a high temperature with the desired thickness

According to the physical phenomenon of the SLS process, there are several phenomena occurring along the process such as;

- Heat generation and transfer; as the powder bed is heated and then the sintered part is cooled down,

- Evolution of the microstructure; where the phase changes with porosity evolution,

- Effects of the fluid; where the molten binder flows to the solid lattice,

- Mechanical issues; e.g. thermal strains during the cooling stage which may cause stresses and distortions.

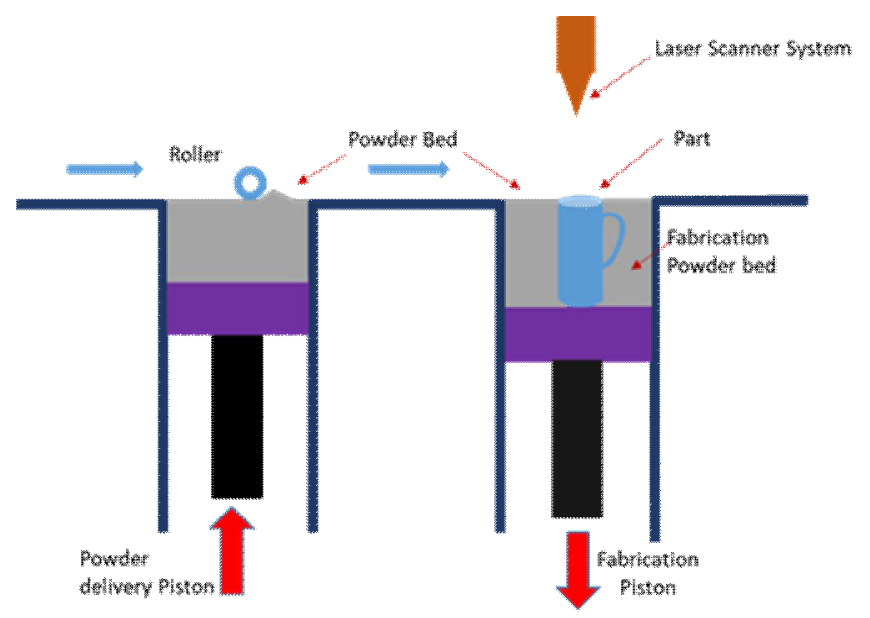

Figure 1: Schematic layout of a the SLS machine process

The SLS technology has received increasing attention in biomedical field. In recent years, SLS been used mostly in orthopaedics and tissue engineering areas and with the use of scanning equipment's such as CT and MRI scan data to generate biomedical models for part formation. The speed of SLS process, the functional properties and characterization of the manufactured part have allowed SLS to be recognized as an important fabrication method in comparison to conventional manufacturing processes or other $3 \mathrm{D}$ printing methods. This review is based on extensive review of several journal paper published in SLS in respect to categories such as application and material developed to biomedical engineering. 


\section{SLS PROCESS PRAMETERS}

There are several process parameters of the SLS process that affect the mechanical properties, surface characteristics and the quality of the fabricated part. In fabricating the part, the characteristics such as part density, microstructure and the mechanical strength are mainly influenced by a combination of process parameter settings. Therefore, the process parameter and their characterization are further divided into three different categories i.e. Laser, Scan and Machine Parameters. There are different types of lasers used in SLS for part fabrication, such as $\mathrm{CO} 2$ or $\mathrm{Nd}$ : YAG laser beam to scan the successive powder layers to create a three-dimensional part [3]. The ideal laser energy density can be achieved by adapting the laser power and the scan speed. Different type of the lasers has high influence on the strengthening of the powder particles. The absorption of the laser on different materials depends on the wavelength of the laser. As per the use of $\mathrm{CO} 2$ lasers on the polymers and oxy-ceramics, they have high absorption of the laser wavelength of $10.6 \mu \mathrm{m}$ [4]. When the laser power and the scanning speed increases or decreases, the fabricated part will be porous or denser. However, if the energy density is higher, it will lead to melt the binder, which will reduce the delamination of the material and the density of the fabricated part is increased [5]. Nonetheless, higher energy density can affect the mechanical properties and may lead to inaccurate dimensions. Similarly shorter laser exposure time on material may lead to difficulty in fabrication of high density part. The laser power is one of important parameters since other parameters will depend on its settings

Previous studies carried out suggests that the energy during the laser scanning, that has been delivered on the surface of the powder, is the main factor in determining the quality of the part fabricated in SLS [5]. In the scanning path, there are two main factors on which the performance of the final sintered part depends i.e. generation and the control of the scanning path. Since every layer is made by the laser sintering along a linear scanning path, the long straight lines of the sintering path may result in shrinkage and will affect the accuracy of the sintered parts. Furthermore it will affect the mechanical strength due to poor geometric relationships [6]. The path should be self-avoiding, and, in the layer, every point must be sintered but not more than one time or else the over sintered point will be visible. The scanning path should be simple and convenient in the order to gain real time control.

The scanning speed of the laser beam is same as the point distance divided by the exposure time. The scanning speed plays an important role on the sintering process. As the scanning speed of the laser is decreased, denser parts can be achieved and is caused by the longer interaction time between the beam and the powder [6]. Furthermore, if the scanning speed of the laser is increased; the energy transferred to the material will be less which will result in less sintering and higher rate of porosity. Therefore, if the energy delivered to the power bed is increased, there will be better melting of the powder which will increase liquid phase to flow and fill the void between particles thus leading to denser structure.

\section{BIO MATERIALS USED IN SLS PROCESS}

Selective Laser Sintering has been extensively used to process a variety of bioactive and biodegradable polymers and composites. They have been used for biomedical applications in SLS for the formation of parts, structures or medical devices [7]. Many of these thermoplastic polymers are available from the suppliers of the SLS systems or are available commercially. The main features of these bio-active materials that makes them a great contender for biomedical application include high thermal stability, fatigue, temperature and abrasion resistance, low moisture absorption and low creep and friction with high strength, porosity, light weight, providing great flexibility, incredible mechanical properties and excellent surface resolution [8].

The temperature transition point and the melting temperature are some of the important factors to consider when describing the type of polymers [8]. The physical, mechanical and chemical properties of end product are directly related to this initial understanding, which is what dictates the type of system setting required. The common type of plastic for laser-based fabrication systems such as SLS is the thermoplastic which either comes under amorphous or semi-crystalline polymer [9]. It is based on how the molecular structure is arranged for each type and the temperature it requires for any changes to occur. The glass transition temperature and the melting temperature are used to categorise the changes during the fabrication process while using the thermoplastic polymers [9]. The glass transition temperature occurs earlier in the process of melting. It is usually at the stage of the glass transition temperature when the polymer material starts to lose the elastic modulus and then further heating to higher temperatures will induce it to melting temperature. It is this high temperature which causes the changes at the molecular level to the chain structure to form a very high viscus looking liquid [9].

Polycaprolactone (PCL) and a number of other, cellulose natural and synthesized polymers have been studied for considerable amount of time due to the advantages they exhibit. Using SLS for the fabrication of tissue engineering scaffold, cellulose has been used on a reasonably large number of times for its natural abundance. PCL being a synthetic polymer with some great characteristics and high thermal stability has been known to be used on application of tissues engineering and cartilage repair process [10,11].This synthetic polymer has great bioresorbability; biodegradation 
period is approximately around two years and is very biocompatible. The polymers that possess these qualities are not in great abundance and hence a composite is an option that some of the new studies are considering.

The mechanical properties of ceramics and polymers are very different individually but collectively they could Combining them can lead to new properties that can be used for the fabrication of some important bioactive parts.

Various studies have been conducted in past with this approach in fabricating scaffolds containing ceramic and polymer. Some studies have also been conducted where SLS has been used to sinter Hydroxyapatite that had been modified with polymeric binder coating. Recent studies suggest that polyethertherketone (PEEK) has exceptional chemical and mechanical properties for a synthetic polymer $[12,13]$. PEEK is considered to be a thermoplastic with semi-crystalline molecular chain structure with relatively high modulus of elasticity (3.6GPa) and a tensile strength of 100MPa. PEEK and Hydroxyapatite (HA) show great bonding, since the glass transition temperature of PEEK is about $143{ }^{\circ} \mathrm{C}$ and its melting point is greatly lower than that of (HA), and it forms good bond within the SLS sintered matrix [14]. Calcium phosphate (CP)/poly(hydroxybutyrate-co-hydroxyvalerate) (PHBV) also carbonated hydroxyapatite (CHA)/poly (L-lactic acid) (PLLA) has been found to have some profound biological properties. Such composites have been used in the tissue scaffold applications. These composites have been previously found to have been used in porous scaffold applications and have been fabricated by the use of SLS.
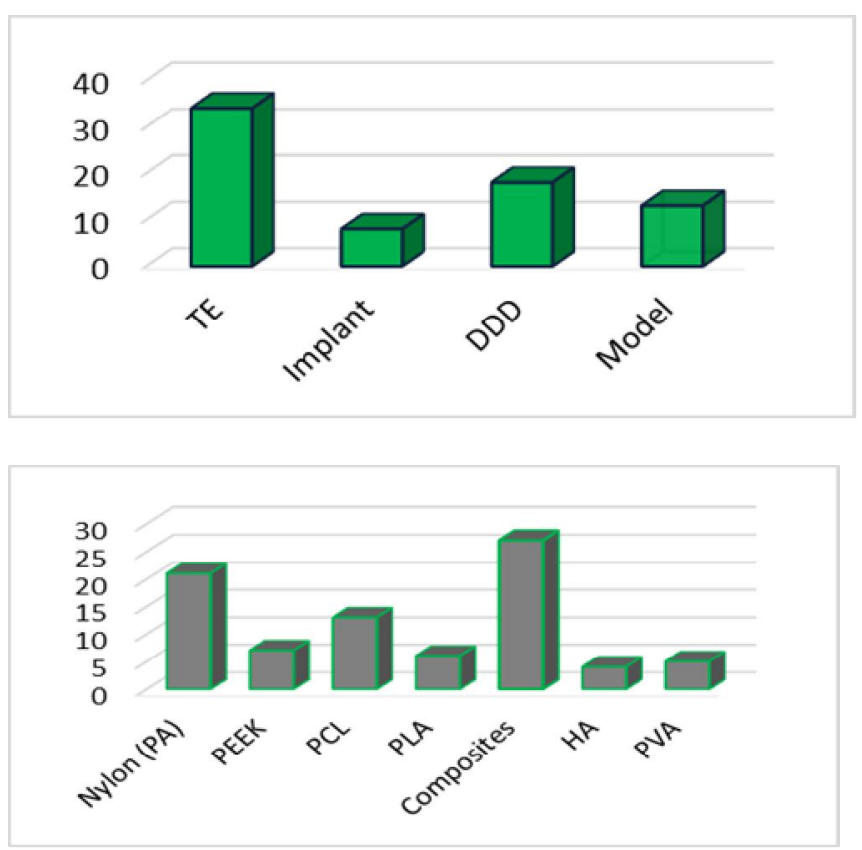

Figure 2: The number of publications in SLS in biomedical engineering (a) in different applications and (b) using the types of materials (up until Oct 2018)
Ceramics have been used for tissue engineering for some time but the mode that has been accepted for the processing has been either extraction based which does have great compressive strength but lacks the feasibility when it comes to complex design such as internal trabecular type of structure. Ceramics can be sintered using SLS systems in two forms, it can be either sintered in slurry form or in powder form, but slurry has slight advantage over the powder in terms of cracking $[15,16]$. The problem with powder form is that due to the high heat, the fabricated part undergoes high thermal stress which induces cracking of the part. The density at which the bed is packed is lower as well hence the part is easily subjected to high thermal stress.

Some of the other very useful ceramic scaffold that has been fabricated and still is of interest over the last two decade is the calcium silicate (CS, CaSiO3) [16]. Calcium silicate with hydroxyapatite (HA) as bioactive ceramics are used on many occasions for tissues engineering purposes. The SLS process using bioactive and biocompatible minerals for human skeletal systems such as teeth and bones has been of great interest. The commonly used ceramics for the purpose of tissue engineering of bone scaffolds are Hydroxyapatite (HA,Ca10(PO4)6(OH)2, Calcium Phosphate $(\mathrm{CP})$ and CS, which can be used to form interconnected pore structured scaffold using SLS and when soaked in simulated bodily fluid (SBF) they have the ability to form apatite layer on its surface [17]. Some of the other ceramics that are used broadly are glass ceramics and crystalline-ceramics, where the glass ceramic has been observed to have increased mechanical properties $[18,19]$. The dissolution products of these bio-active glasses have been explored and been found to be beneficial with the regulation of gene that is responsible for the repair of bone tissues, which increases the formation of the bone and helps the repair process. The glass ceramic has been found to have considerable advantage over other ceramics such as HA.

Cellulose derived biomaterials have been used for some time for various biomedical application due to their biocompatibility. Cellulose composites and polymer derived has been used for the fabrication of control drug release mechanism such as drug delivery scaffolds that are sintered from starch cellulose and cellulose acetate using the SLS system. The cellulose that has been used more often is the Mater-Bi starch-cellulose Grade Y101U and it exhibited the glass transition temperature is $102^{\circ} \mathrm{C}$ which indicates an amorphous material.

\section{PROPERTIES AND APPLICATIONS OF SLS FABRICATED COMPONENTS}

Major applications of SLS in biomedical engineering include tissue engineering scaffolds, drug delivery devices (DDD), 
implants and prostheses, and anatomical models. Figure 2(a) shows the approximate number of publications as obtained from Google search (up until Oct 2018) in these four major areas of SLS applications. Figure 2(b) shows the approximate number of publications related to biomaterials used in SLS process by researchers as searched from Google (up until Oct 2018). SLS parameters play an important role in controlling the strength of the fabricated structure. SLS fabricated parts also has challenges of biocompatibility. Application in tissue scaffolding, drug delivery devices and implants requires close control of architectural properties such as pore size permeability polymer hydrophobicity, and availability of bioactive binder sites for cell adhesions [20,21]. Control of the architecture assists in achieving the structure that is accepted for its purpose. For tissue engineering application, it is desirable to achieve a high compressive strength for an interconnected pore structure that would be used as a tissues scaffold.

For Drug Delivery Devices (DDD), various SLS process parameters play key role to impart diffusion properties for the material being used for fabrication of the structure, considering the complexity of the design of the part [22]. The laser setting is extremely important as it will determine the densification of the structure. For a DDD, the drug loading and releasing is a very important function. To investigate the function of the newly fabricated part, methylene blue is used to observe the release time. This could be either done in water or in a phosphate buffer solution. The release of methylene blue in the phosphate buffer solution (PBS) can be quantified using UV spectrometer The PBS has very close $\mathrm{pH}$ level to human blood which is around 7.4. The release is compared with the calibrated value indicating the release period. Temperature at which the release test is conducted is an important parameter to consider as well. The microstructure needs to be examined for porous canals, porous matrix and loose powder.

For Tissue Engineering application, the scaffold macro-architecture will have great influence on how the scaffolds will function efficiently with respect to both the stability of scaffold and with the proliferation and interaction with the cell. The scaffold is usually developed using a unit cell on a CAD system then it is amplified, multiplied and multiple units are combined to form the structure. During this process its stiffness, porosity, tensile, compressive and bending properties are analyzed so that the complete structure is suitable for the application [23]. One very important feature is vascularization, which is needed for the bulk growth of tissues. Some new approaches have been taken, such as use of thin porous membranes, which is alternated between layers for 3D scaffold pattern. Shrinkage and non-uniformity due to shrinkage could also cause issues. Micro-structural analysis is done by defining the region of interest and using $\mu \mathrm{CT}$ scanner. The porosity is calculated to reassure the initial requirements of the structure. Mechanical properties such as compressive strength and the compressive modulus as well as the strain levels are compared to the CAD models. Another mode of confirmation of the newly fabricated structure is done using the FEA image-based analysis, where the structure is reviewed for all the properties and characteristics which have been the initial requirements. Cell seeding and implantation is then done after all properties satisfy the parameters required.

Implants made from biocompatible materials in SLS need to be able to resist wear and be able to sustain cyclic and static loading for most of human body parts as indicated by some researchers [24]. Some of the properties that are required and should be tested prior to the implants being classified as suitable for the application are: ductility, interconnected porosity, high density with low weight and durability, high strength with great stuffiness, bone and blood compatibility [24]. Deterioration, temperature resistance and non-corrosive features are also important when considering for an implant to be part of human body. Some of common non-metallic materials for bone implants are known to be Nylon, PA, PS, and PC. For the testing of strength, the fabricated structure is subjected to tensile, compressive and fatigue testing using universal testing machine (UTM). Similarly the $\mathrm{pH}$ and blood compatibility test is conducted in buffer type solutions. Only after such comprehensive tests, the implant is considered beneficiary of the recipient. The cellular or tissue response is tested prior implantation.

Anatomical models are used for medical training and education. These models are usually fabricated either using binder jet (BJ) process or SLS process as they produce much better quality of models with detailed features and also provide strength for it to be used for multiple occasions. The powder used for such models is usually polyamide, nylon and glass beads to give required properties for the finished product. The anatomical parts that are fabricated for medical training or preparation prior to some complex operative procedure are required to be very close in the properties as the actual anatomical features, since the training of the procedure is done on the model and it is important to keep it as real as possible [25]. Some examples of these detailed model structures are Henle's spine, tympanomastoid suture and temporal bone [26]. Using SLS, the main advantages are mechanical strength, temperature resistant, build speed and the layer thickness. The morphology of the structure is an important feature since it provides surface details of the anatomy. The model feature and properties are usually tested for its durability, compressive, tensile strength, and similarly for other application using UTM and microscopy. 


\section{SUMMARY AND FUTURE WORK}

This brief overview of selective laser sintering additive manufacturing has identified the current trend of this technology in biomedical engineering applications and the range of biomaterials used in this field. The SLS process has attracted growing attention in this sector especially in tissue engineering and development of new composites for biomedical applications. The flexibility to achieve most difficult structure by the use of CT scan and CAD data and then fabricate the structure layer by layer makes SLS a popular fast growing method of 3D printing. The energy required on surface for the formation of the structure is not as much in comparative to other laser based 3D printing methods and the process can be controlled to achieve desired strength and morphological features. The advantages of this method of additive manufacturing outlay the limitations. It has been observed that SLS has great potential in the biomedical field with its ability to sinter most of the powder base materials that are available and without the need for expensive post processing. The product is ready in reasonably fast fabrication time. This ability to fabricate from MRI and CT scanned data and at a speed that has potential to save time in surgical procedure shows it has great prospects in biomedical applications. The limitation at present is the much-needed range of various biocompatible materials in powder forms and the cost of these powders need to be more affordable. The composite mix of materials needs more binders with various properties that could help maintain the desired characteristics.

\section{REFERENCES}

1. Pilipović, A., Drstvenšek, I. \& Šercer, M. 2014. Mathematical Model for the Selection of Processing Parameters in Selective Laser Sintering of Polymer Products. Advances in Mechanical Engineering, 6, 648562.

https://doi.org/10.1155/2014/648562

2. Shuai, C., Gao, C., Nie, Y., Hu, H., Zhou, Y. \& Peng, S. 2011. Structure and properties of nano-hydroxypatite scaffolds for bone tissue engineering with a selective laser sintering system. Nanotechnology, 22, 285703.

3. Yang J, Bin H, Zhang X \& Liu Z. 2003. Fractal scanning path generation and control system for selective laser sintering (SLS). International Journal of Machine Tools and Manufacture, 43, 293-300.

4. Kumar S. 2003. Selective laser sintering: a qualitative and objective approach. Jom, 55, 43-47. https://doi.org/10.1007/s11837-003-0175-y

5. Das S, Hollister J , Flanagan C, Adewunmi A, Bark, K, Chen, C, Ramaswamy K, Rose D \& Widjaja E 2003. Freeform fabrication of Nylon-6 tissue engineering scaffolds. Rapid Prototyping Journal, 9, 43-49.

6. Santos E C, Shiomi M, Osakada K \& Laoui T. 2006. Rapid manufacturing of metal components by laser forming. International Journal of Machine Tools and Manufacture, 46, 1459-1468.

7. Edith Wiria F, Sudarmadji N, Fai Leong K, Kai Chua C, Wei Chng E \& Chai Chan C. 2010. Selective laser sintering adaptation tools for cost effective fabrication of biomedical prototypes. Rapid Prototyping Journal, 16, 90-99. https://doi.org/10.1108/13552541011025816

8. Peltola S M, Melchels F P, Grijpma D W \& Kellomäki M. 2008. A review of rapid prototyping techniques for tissue engineering purposes. Annals of medicine, 40, 268-280.S J \& Das S. 2006.

9. Drummer D, Rietzel D \& Kühnlein F. 2010. Development of a characterization approach for the sintering behavior of new thermoplastics for selective laser sintering. Physics Procedia, 5, 533-542.

10. Chen H, Lee Y, Shyu V, Chen C, Chen T \& Chen P. 2014. Surface modification of polycaprolactone scaffolds fabricated via selective laser sintering for cartilage tissue engineering. Materials Science and Engineering: C, 40, 389-397.

11. Yeong W, Sudarmadji, N, Yu H, Chua C, Leong K, Venkatraman S, Boey Y \& Tan L. 2010. Porous polycaprolactone scaffold for cardiac tissue engineering fabricated by selective laser sintering. Acta biomaterialia, 6, 2028-2034.

12. Mazzoli A. 2013. Selective laser sintering in biomedical engineering. Medical \& biological engineering \& computing, 51, 245-256. https://doi.org/10.1007/s11517-012-1001-x

13. Shuai C, Cao Y, Gao C, Feng P, Xiao T \& Peng S. 2015. Hydroxyapatite whisker reinforced $63 \mathrm{~s}$ glass scaffolds for bone tissue engineering. BioMed research international, 2015 Products. Advances in Mechanical Engineering, 6, 648562.

14. Tan, K. H., Chua, C. K., Leong, K. F., Cheah, C. M., Cheang, P., Abu Bakar, M. S. \& Cha, S. W. 2003. Scaffold development using selective laser sintering of polyetheretherketone-hydroxyapatite biocomposite blends. Biomaterials, 24, 3115-3123.

15. Mühler, T., Gomes, C., Ascheri, M. E., Nicolaides, D., Heinrich, J. G. \& Günster, J. 2015. Slurry-based powder beds for the selective laser sintering of silicate ceramics. Journal of Ceramic Science and Technology, 6, 113-118.

16. Kumaresan T, Gandhinathan R, Ramu M, Ananthasubramanian M \& Pradheepa K B 2016. Design, analysis and fabrication of polyamide/hydroxyapatite porous structured scaffold using selective laser sintering method for bio-medical applications. Journal of mechanical science and technology, 30, 5305-5312.

17. Chua C, Leong K, Tan K, Wiria F \& Cheah C. 2004. Development of tissue scaffolds using selective laser sintering of polyvinyl alcohol/hydroxyapatite biocomposite for craniofacial and joint defects 
18. Deckers, J., Vleugels, J. \& Kruth, J. P. 2014. Additive manufacturing of ceramics: A review. Journal of Ceramic Science and Technology, 5, 245-260.

19. Liu, J., Zhang, B., Yan, C. \& Shi, Y. 2010. The effect of processing parameters on characteristics of selective laser sintering dental glass-ceramic powder. Rapid Prototyping Journal, 16, 138-145. https://doi.org/10.1108/13552541011025861

20. Barron V, Flaherty N, Cahill S, Tyndyk M, Mchugh P. \& Lohfeld S. 2010. A method to fabricate small features on scaffolds for tissue engineering via selective laser sintering. Journal of Biomedical Science and Engineering. et al., 2010

21. Peltola, S. M., Melchels, F. P. W., Grijpma, D. W. \& Kellomäki, M. 2008. A review of rapid prototyping techniques for tissue engineering purposes. Annals of Medicine, 40, 268-280.

22. Leong K, Chua C \& Gui W. 2006. Building porous biopolymeric microstructures for controlled drug delivery devices using selective laser sintering. The International Journal of Advanced Manufacturing Technology, 31, 483-489.

23. Eshraghi, S. \& Das, S. 2012. Micromechanical finite-element modeling and experimental characterization of the compressive mechanical properties of polycaprolactone-hydroxyapatite composite scaffolds prepared by selective laser sintering for bone tissue engineering. Acta Biomaterialia, 8, 3138-3143.

https://doi.org/10.1016/j.actbio.2012.04.022

24. Tiwari SK, Pande S, Agrawal S, Bobade SM. Selection of selective laser sintering materials for different applications. Rapid Prototyping Journal. 2015;21(6):630-48.

25. Mori, K., Yamamoto, T., Oyama, K. \& Nakao, Y. 2009. Modification of three-dimensional prototype temporal bone model for training in skull-base surgery. Neurosurgical Review, 32, 233-239. https://doi.org/10.1007/s10143-008-0177-x

26. Torres K, Staśkiewicz G, Snieżyński M, Drop A. \& Maciejewski R. Application of rapid prototyping techniques for modelling of anatomical structures in medical training and education. Folia Morphologica, 70, 1-4, 2011. 